\title{
Study on Pre-Crack Marble Brazilian Disc Crack with SHPB Experiment and Numerical Simulation
}

\author{
Jiang-Feng $\mathrm{Ma}^{1, \mathrm{a}}$, Jing-Chun $\mathrm{Xu}^{2, \mathrm{~b}}$, Xiu-Li Zhang ${ }^{3, \mathrm{c}}$ and Yao Rong ${ }^{4, \mathrm{a}}$ \\ ${ }^{1,4}$ Jiangxi transportation institute, Nanchang 330200, China \\ ${ }^{2}$ Ganzhou expressway company, Ganzhou 334100, China \\ ${ }^{3}$ Institute of Rock and Soil Mechanics, Chinese Academy of Sciences, Wuhan 430071, China \\ amj55666@163.com
}

\begin{abstract}
Keywords: crack propagation, numerical simulation, SHPB experiment, pre-crack Brazilian disc. Abstract. We presented a new two-dimensional crack criterion for DDA (Discontinuous Deformation Analysis) method to simulate the failure of pre-crack Brazilian disc marble specimens under dynamic loading. The Split Hopkinson Pressure Bar (SHPB) equipment was used to impact the specimen and check the applicability and validity of the improved theoretical fracture criteria. The mechanism of crack propagation and the influence of pre-crack on crack process are explored through experiment and numerical simulation. It is concluded that the simulation results reproduce the whole fracture process, i.e., the initiation, propagation and failure of the specimen under dynamic loading condition, agreeing favorably with those results obtained from SHPB tests. The results show that: (1) the pre-crack specimen cracks from pre-crack tips firstly, and then expands to the sides in opposite directions, eventually splitting to two separate parts; (2) the fracture mode of pre-crack rock specimen is tensile failure, and the tensile stress of crack tip is the direct driving force leading to crack propagation.
\end{abstract}

\section{Introduction}

Explosion is the most common method in tunnel engineering, mining engineering and rock slope excavation. How to accurately simulate and manipulate the deformation and crack propagation of rocks under blast impact loading is one of the hottest and most difficult issues for geoengineers. Because of the heterogeneity and anisotropy of intact rocks, the crack propagation process induced by explosion is very complicated. Moreover, in reality, most rocks are cut by joints and cracks that interact with each other, which make the crack process of jointed rocks more complicated under dynamic loading. With the development and progress of the experimental equipment and techniques, people make a large number of laboratory and field experiments to study the dynamic mechanical properties, failure criteria and constitutive relation of rocks under impact loading. Experiment with Split Hopkinson Pressure Bar (SHPB) is a necessary means in studying the mechanical response of rock materials under high strain rate and scholars have gained great achievements in this aspect. The effect of dynamic loading on the stress-strain curve and cracking of sandstone was studied by using SHPB experiment [1]. The influence of loading rate on fracture toughness and micro cracking mechanism was analyzed [2]. [3-5] compared the tests of marble in various shapes by using SHPB to confirm the dynamic fracture toughness and put forward the corresponding formula. However, in the past experiments, more attentions were paid to the relationship between the mechanical parameters including elastic modulus, fracture toughness and strain rate of intact rocks under dynamic loading. In the numerical simulation, the stress distribution of Brazilian disc in SHPB experiment has been simulated by using finite element method [6]. The dynamic fracture process of granite had been simulated under FEM/DEM model, and the simulation results of the major crack were good [7]. [8] proposed the mesoscale model of concrete material and simulated the crack propagation and fracture under high strain rate. [9] simulated the cleavage of Brazilian disc specimen under static and dynamic loadings by using RFPA. But the form of the stress wave is difficult to implement in reality. Literatures $[6,8,9]$ studied impact problem from the numerical simulation angle, but there were no 
experimental results to be compared with. Most numerical simulation models were based on intact rock specimen and few researchers studied the simulation of the dynamic fracture process with pre-cracks.

By taking pre-crack Brazilian disc specimen as the research object and combining with laboratory experiments and numerical simulations, the real crack propagation and fracture process of rock specimen under blast impact loading by SHPB is simulated by using DDARF (Discontinuous Deformation Analysis for Rock Failure) program. The crack propagating paths, causes and the spring back phenomenon of the incident bar are shown clearly. The micro failure criteria of rocks under blast impact loading and the effect of pre-crack on crack propagation are investigated. The dynamic tensile strength and the final failure pattern of marble specimen are obtained. The simulation results and the experiment results are in good agreement, and the correctness of dynamic crack criteria is verified to some extent.

\section{SHPB Experiment}

Instrument Introduction. The schematic diagram of the SHPB equipment is shown in Fig. 1. SHPB is mainly made up of launcher and experiment device. The launcher includes the bore, barrel, control box and gas cylinder, while the equipment device includes incident bar, transmission bar, buffer bar and measurement system. The diameters of the three steel bars are all $50 \mathrm{~mm}$ and the lengths of incident bar, transmission bar, and buffer bar are $2500 \mathrm{~mm}, 2500 \mathrm{~mm}$ and $1000 \mathrm{~mm}$, respectively. The elastic modulus is $210 \mathrm{GPa}$ and the density is $7800 \mathrm{~kg} / \mathrm{m} 3$. The theoretical and measured velocities are $5189 \mathrm{~m} / \mathrm{s}$ and $5124 \mathrm{~m} / \mathrm{s}$, respectively. The projectile is driven by the impact of nitrogen. The impact velocity is measured by the laser velocimetry system made up of the time interval instrument. A $4 \mathrm{~mm}$ by $4 \mathrm{~mm}$ quadrate natural rubber is pasted on the center of the incident bar to adjust the form of stress waves. When the projectile impacts the incident bar co-axially and after the adjustment of the pulse shaper, the incident stress wave will come into being on the incident bar and act on the specimen. Then the specimen will deform in high speed. In the meantime, the reflected stress wave in reverse direction and transmission stress wave in forward direction will occur. The stress wave is recorded by the strain gauge pasted on the bar. And the stress wave is saved and displayed in the oscilloscope.

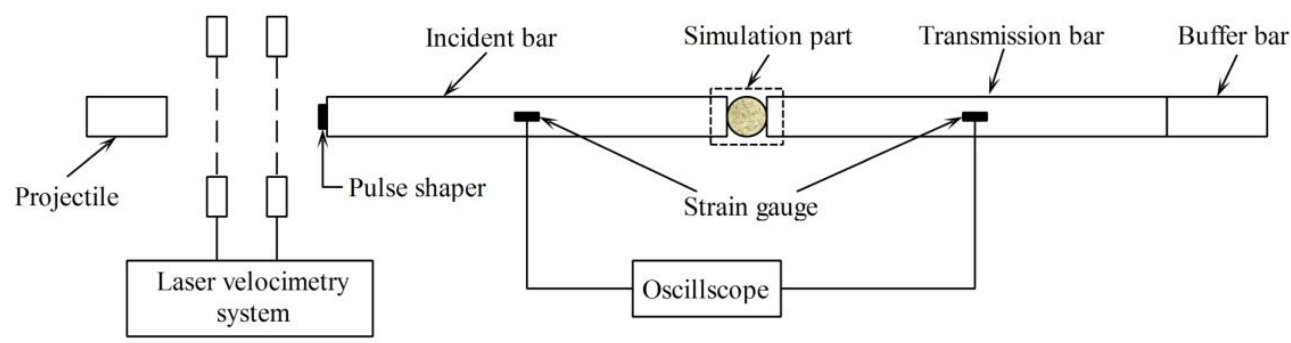

Fig. 1 Sketch of SHPB experimental setup

Experiment Scheme. In the experiment, the Brazilian disc specimens with pre-cracks are made of white marble. The mechanical properties of the rock material are as follows: elastic modulus $\mathrm{E}=16$ GPa; poison's ratio $\mu=0.3$; density $\rho=2670 \mathrm{~kg} / \mathrm{m} 3$; internal friction angle $\varphi=30^{\circ}$. The diameter and thickness of the specimen are $50 \mathrm{~mm}$ and $25 \mathrm{~mm}$, respectively. The pre-crack is at the center of the Brazilian disc and the length and width are respectively $12 \mathrm{~mm}$ and $2 \mathrm{~mm}$. The pre-crack specimen is shown in Fig. 2. 


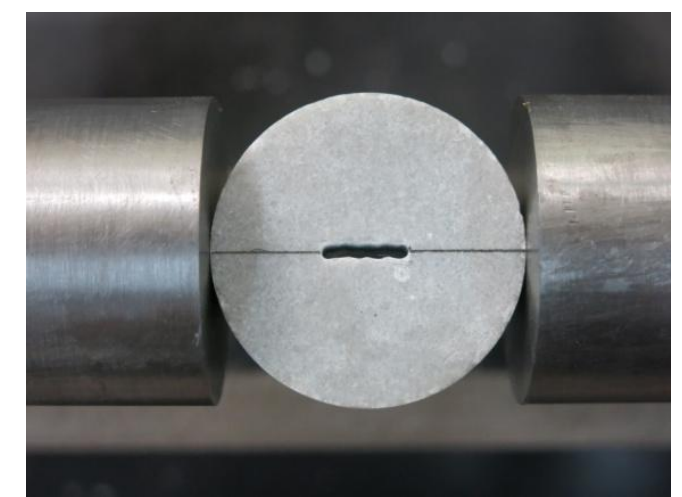

Fig. 2 Photograph of the pre-crack specimen

The specimen is placed horizontally in the middle of the incident and transmission bar along diameter direction. The positions contacted to the incident and transmission bar are smeared by Vaseline to eliminate the effect of frictional force in impact process. The impact speed is controlled by setting different pressure values. The higher the air pressure is, the quicker the impact speed is, meanwhile, the bigger the force acted on the specimen is. In this experiment, the air pressure is set to $0.05 \mathrm{MPa}$. Based on one-dimensional stress wave theory, the stress acted on the specimen by the right end of the incident bar can be obtained from Eq. (1).

$$
\sigma(t)=E\left[\varepsilon_{i}(t)+\varepsilon_{r}(t)\right]
$$

In this equation, $\sigma(\mathrm{t})$ is the stress acted on the specimen by the incident bar and $\mathrm{E}$ is elastic modulus of the bar. $\varepsilon_{\mathrm{i}}(\mathrm{t})$ and $\varepsilon_{\mathrm{r}}(\mathrm{t})$ are respectively incident and reflection wave strain.

\section{Numerical simulation}

Numerical Model. Comparing the size of rock specimen with the incident and transmission bar, they are not in the same magnitude. If the simulation takes the real size, the specimen is comparatively small, which goes against the observation of crack propagation. According to Saint-Venant's Principle, we know that stress wave form is more stable on certain length scales. So in the numerical simulation, we take the end faces of the incident and transmission bar. The geometry size and loading boundary are shown in Fig. 3. The incident and transmission bar are respectively on the left and right side of the specimen and their lengths are both $5 \mathrm{~mm}$. The stress wave is applied from the incident bar and the transmission bar is fixed.

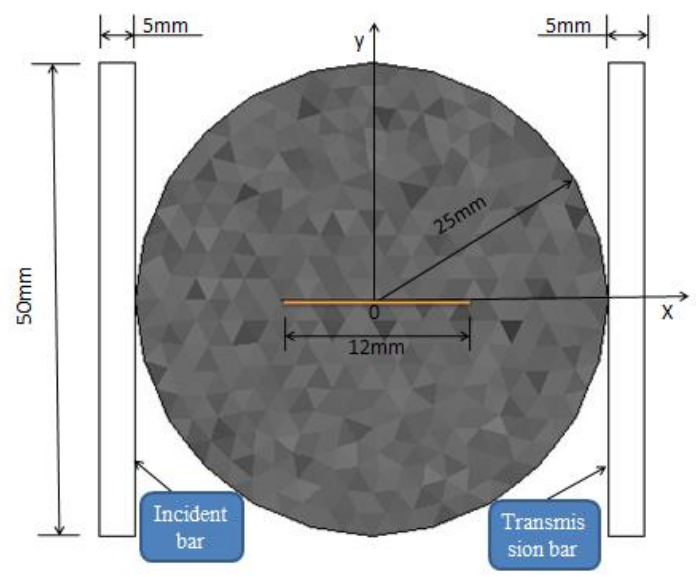

Fig. 3. The calculation model of pre-crack specimen

Simulation Results and Analysis. The crack propagation, displacement contours, and the minimum principle stresses of pre-crack specimen in different time steps are shown in Fig. 4. At initial stage, with the loading of incident stress wave, deformation and displacement that are symmetrical about $\mathrm{x}$ axis appear in the specimen and tensile stress at crack tips increases gradually. When tensile stress reaches failure strength, the bond spring between two blocks will fail and 
pre-crack initiates from the crack tips. After crack initiation, the crack propagates to both sides along diameter direction. In the crack propagating process, tensile stress near the new crack tips is always very large. In the end, crack coalescences and the specimen is split into two separate parts. The incident bar produces displacement along the negative direction of $\mathrm{x}$ axis. The simulation results and experimental results are compared in Fig.5.
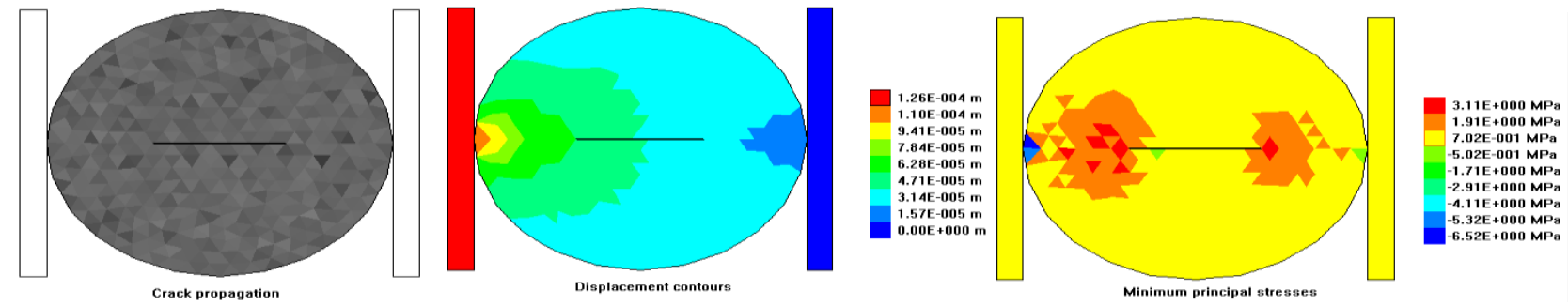

(a) $10 \mathrm{step}(157 \mu \mathrm{s})$
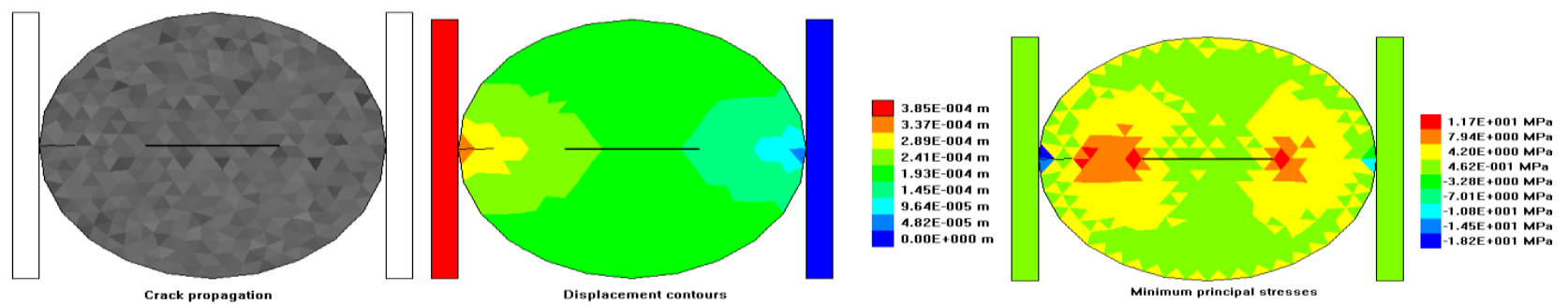

(b) 20 step $(227 \mu \mathrm{s})$
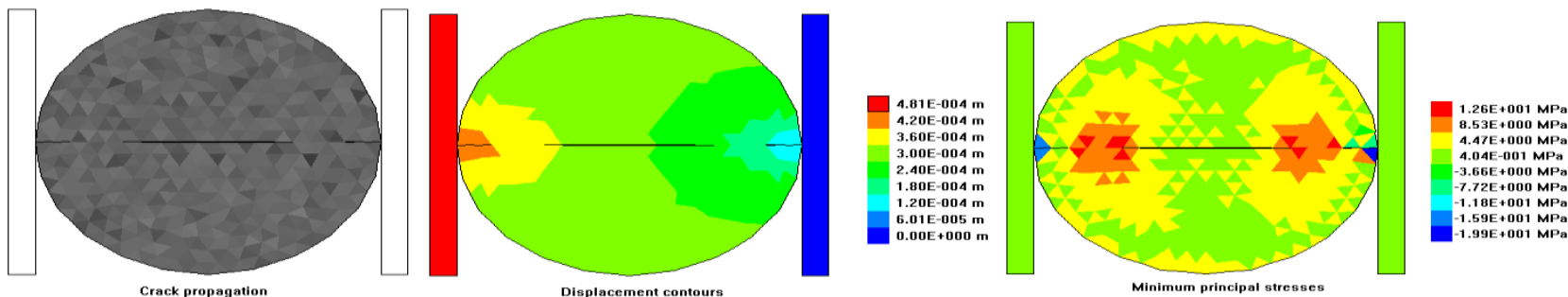

(c) $25 \mathrm{step}(328 \mu \mathrm{s})$
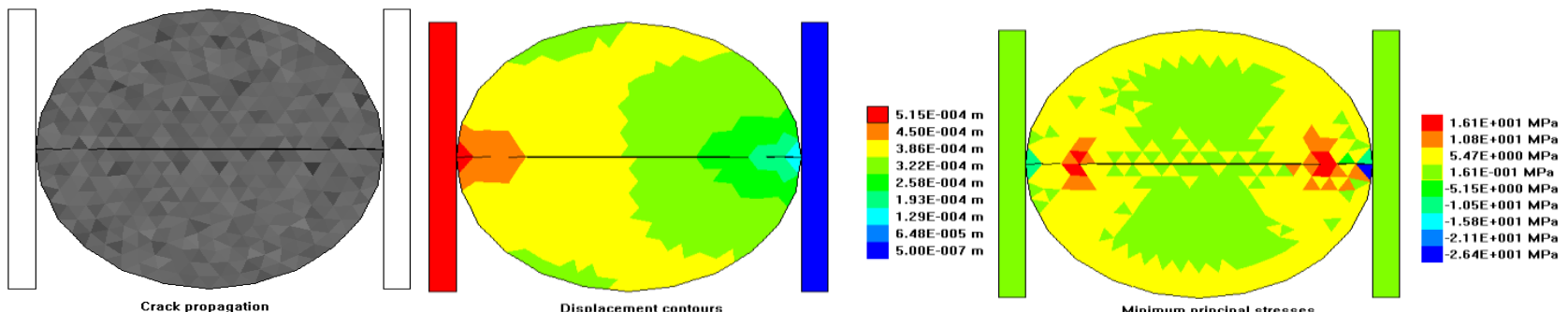

(d) $30 \mathrm{step}(373 \mu \mathrm{s})$
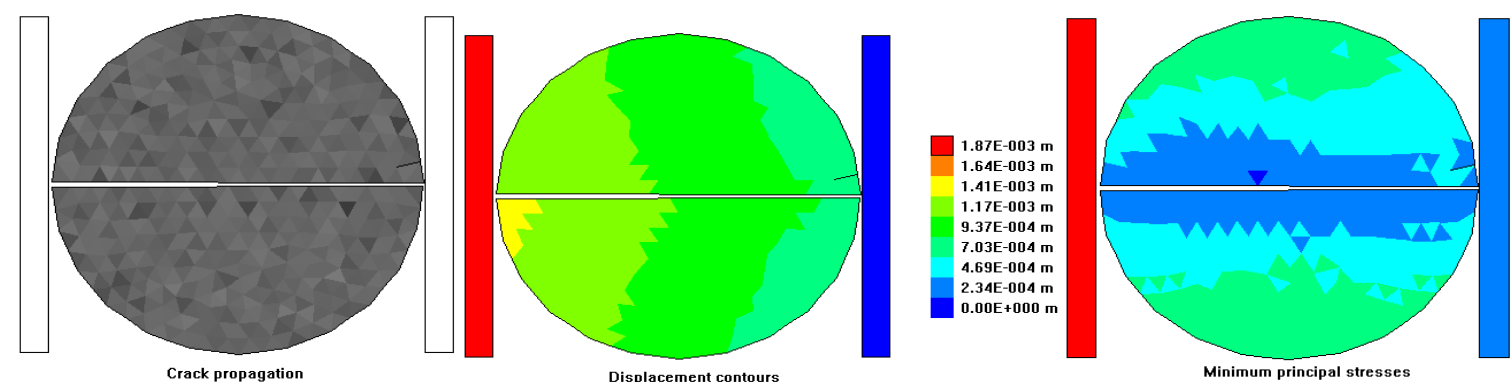

2.07E+000 MP

$1.52 \mathrm{E}+000 \mathrm{MPa}$

$1.52 \mathrm{E}+000 \mathrm{MPa}$

9.78E-001 MPa

$7.05 \mathrm{E}-001 \mathrm{MPa}$
$4.33 \mathrm{E}-001 \mathrm{MPa}$

1.61E-001 MP

(e) 70 step $(793 \mu \mathrm{s})$

Fig. 4The crack propagation, displacement contours, and the minimum principle stresses of pre-crack specimen in different time steps:(a) 10 step (157us), (b) 20 step (227us), (c) 25 step (328us), (d) 30 step (373us), and (e) 70 step 

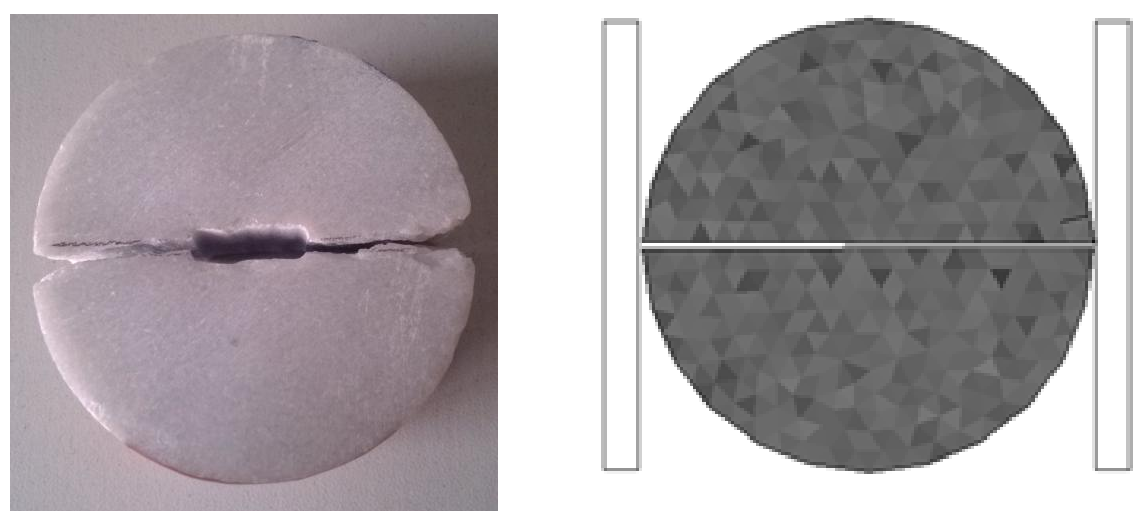

Fig. 5 The experimental results and simulation results

\section{Conclusions and Discussions}

The real crack propagation process of pre-crack Brazilian disc specimen under blast impact loading imposed by SHPB is simulated by using DDARF program. The cause of crack propagation is studied and the validity of the improved criteria is confirmed. The following conclusions are obtained:

(1) Simulation results and experimental results are in good agreement. Simulation results based on improved criteria have remarkable effect and applicability in simulating dynamic fracture of jointed rock.

(2) Under the impact loading, pre-crack Brazilian disc first initiates at pre-crack tips and then propagates to both sides along radial loading direction until coalescence rupture. The tensile stress at crack tips is the main cause that induces the failure of the rock.

The fracture process of rock is a complicated. The propagation direction, failure mode, last failure form are not only closely related to the incident wave amplitude, loading rate but also to the lithology, interaction between cracks, material heterogeneity, self-micro defects and so on. There is some randomness in microscope, and the simulated mode does not necessarily apply to all loading situations. In reality, the angle, length, and quantity of the joints are different. To inquiry the effect of different angle, length, quantity pre-cracks to the fracture of the rock is the emphasis of future work.

\section{Acknowledgements}

This work was financially supported by the Department of Transportation projects of Jiangxi Provincial (2015C0067, 2015C0016), the Key Research Program of the Chinese Academy of Sciences (KZZD-EW-05-03), and the China National Natural Science Foundation (40972201, 51025935).

\section{References}

[1] R. J. Christensen, S. R. Swanson and W. S. Brown, Split-Hopkinson-bar Tests on Rock under Confining Pressure, Exp Mech, 12, Issue 11, 508-513(1972).

[2] Q.B. Zhang and J. Zhao. Effect of loading rate on fracture toughness and failure micromechanisms in marble, Eng Frac Mech, 102, 288-309(2013).

[3] Q.Z. Wang, F. Feng, M. Ni and X.P. Gou, Measurement of mode I and mode II rock dynamic fracture toughness with cracked straight through flattened Brazilian disc impacted by split Hopkinson pressure bar, Eng Frac Mech, 78, 2455-2469(2011).

[4] Q.Z. Wang, W. Li and H.P. Xie, Dynamic split tensile test of Flattened Brazilian Disc of rock with SHPB setup, Mech Mater, 41, 252-260(2009). 
[5] S. Zhang and Q.Z. Wang, Method for determination of dynamic fracture toughness of rock using holed-cracked flattened disk specimen, Chin J Geo Eng, 28, 723-728(2006).

[6] S. Dong, Y. Wang and Y. Xia. A finite element analysis for using Brazilian disk in split Hopkinson pressure bar to investigate dynamic fracture behavior of brittle polymer materials, Poly Test, 25, 943-952(2006).

[7] Mahabadi, B.E. Cottrell and G. Grasselli, An Example of Realistic Modelling of Rock Dynamics Problems: FEM/DEM Simulation of Dynamic Brazilian Test on Barre Granite, Rock Mech Rock Eng, 43, 707-716(2010).

[8] X.Q. Zhou and H. Hao, Mesoscale modelling of concrete tensile failure mechanism at high strain rates, Comput Struct, 86, 2013-2026(2008).

[9] W.C. Zhu, C.A. Tang, Z.P. Huang and M.Z. Pang, Numerical simulation on splitting failure mode of rock under static and dynamic loading, Chin J Rock Mech Eng, 24, 1-7(2005).

[10] G.H. Shi, Block system modeling by discontinuous deformation analysis. Computational Mechanics Publications, Southampton, (1993).

[11]Y.Y. Jiao, X.L. Zhang and J. Zhao, Two-Dimensional DDA Contact Constitutive Model for Simulating Rock Fragmentation, J Eng Mech-ASCE, 138, 199-208(2012).

[12] Y.Y. Jiao, X.L. Zhang, Q.S. Liu and W.Z. Chen. Simulation of rock crack propagation using discontinuous deformation analysis method, Chin J Rock Mech Eng, 26, 682-691(2007).

[13] X.L. Zhang, Y.Y. Jiao and J. Zhao, Simulation of the Failure Process of Jointed Rock, J Cent South Univ, 15, 888-894(2008).

[14] X.L. Zhang and Y.Y. Jiao, Numerical Simulation of Crack Propagation in Three-Point Bending Beams, in: Proceedings of International Young Scholar Symposium on Rock Mechanics 2008(Beijing, China, 2008), pp. 259-262. 